\title{
Implication of the Proton-Deuteron Radiative Capture for Big Bang Nucleosynthesis
}

\author{
L. E. Marcucci, ${ }^{1,2}$ G. Mangano, ${ }^{3}$ A. Kievsky, ${ }^{2}$ and M. Viviani ${ }^{2}$ \\ ${ }^{1}$ Department of Physics, University of Pisa, Largo B. Pontecorvo 3, I-56127 Pisa, Italy \\ ${ }^{2}$ INFN-Pisa, Largo B. Pontecorvo 3, I-56127 Pisa, I-56127 Pisa, Italy \\ ${ }^{3}$ INFN-Napoli, Complesso Univ. Monte S. Angelo, Via Cintia, I-80126 Napoli, Italy
}

(Received 27 October 2015; published 8 March 2016)

\begin{abstract}
The astrophysical $S$ factor for the radiative capture $d(p, \gamma)^{3} \mathrm{He}$ in the energy range of interest for big bang nucleosynthesis $(\mathrm{BBN})$ is calculated using an ab initio approach. The nuclear Hamiltonian retains both two- and three-nucleon interactions - the Argonne $v_{18}$ and the Urbana IX, respectively. Both one- and many-body contributions to the nuclear current operator are included. The former retain for the first time, besides the $1 / m$ leading order contribution ( $m$ is the nucleon mass), also the next-to-leading order term, proportional to $1 / \mathrm{m}^{3}$. The many-body currents are constructed in order to satisfy the current conservation relation with the adopted Hamiltonian model. The hyperspherical harmonics technique is applied to solve the $A=3$ bound and scattering states. Particular attention is paid in this second case in order to obtain, in the energy range of BBN, an uncertainty on the astrophysical $S$ factor of the order or below $\sim 1 \%$. Then, in this energy range, the $S$ factor is found to be $\sim 10 \%$ larger than the currently adopted values. Part of this increase $(1 \%-3 \%)$ is due to the $1 / \mathrm{m}^{3}$ one-body operator, while the remaining is due to the new more accurate scattering wave functions. We have studied the implication of this new determination for the $d(p, \gamma)^{3} \mathrm{He} S$ factor on the deuterium primordial abundance. We find that the predicted theoretical value for ${ }^{2} \mathrm{H} / \mathrm{H}$ is in excellent agreement with its experimental determination, using the most recent determination of the baryon density of the Planck experiment, and with a standard number of relativistic degrees of freedom $N_{\text {eff }}=3.046$ during primordial nucleosynthesis. This calls for a more accurate measurement of the astrophysical $S$ factor in order to confirm the present predictions.
\end{abstract}

DOI: 10.1103/PhysRevLett.116.102501

Introduction.-The radiative capture $d(p, \gamma)^{3} \mathrm{He}$ is a relevant process in many astrophysical environments. For instance, it is the second step in the chain of nuclear reactions which, starting from the proton-proton weak capture, allows stars like our Sun to shine via $p p$ chain. Interest in this reaction is also present in the context of big bang nucleosynthesis (BBN), see, e.g., Ref. [1] and references therein, since it is one of the main processes through which deuterium can be destroyed and thus affects its eventual yield. BBN is a powerful method to test the validity of the cosmological model at the $\mathrm{MeV}$ energy scale. For a given set of values of leading nuclear reaction rates, primordial element abundances depend upon two key cosmological parameters, the energy density in baryons, $\Omega_{b} h^{2}$, and the energy density of relativistic species $\rho_{\text {rel }}$ or, equivalently, the effective neutrino number, $N_{\text {eff }}$, defined as $\rho_{\text {rel }}=\rho_{\gamma}\left(1+\frac{7}{8}\left(\frac{4}{11}\right)^{4 / 3} N_{\text {eff }}\right)$ where $\rho_{\gamma}$ is the cosmic microwave background (CMB) photon energy density. The benchmark value for this parameter with only three active neutrinos contributing is $N_{\text {eff }}=3.046$ [2].

The baryon energy density has been measured with a remarkable precision by CMB anisotropy experiments, so in the minimal $\Lambda \mathrm{CDM}$ scenario light nuclei abundances are basically fixed [3]. Using the most recent determination of the Planck satellite experiment, $\Omega_{b} h^{2}=0.02225 \pm$ 0.00016 [3], and a modified version of the public BBN code PARThENoPE [4], already used in Ref. [5], the primordial deuterium to hydrogen ratio is predicted to be ${ }^{2} \mathrm{H} / \mathrm{H}=(2.61 \pm 0.07) \times 10^{-5}$ (68\% C.L. error). (In the public version of PARTHENOPE the uncertainty on the $d(p, \gamma)^{3} \mathrm{He}$ rate is taken from the analysis of Ref. [6] and is almost a factor of 2 smaller than what we adopt in the present study where, as in Ref. [5], the more conservative 1- $\sigma$ error estimate of Ref. [7] is used. This is the reason for the larger uncertainty quoted on ${ }^{2} \mathrm{H} / \mathrm{H}$. Central values for ${ }^{2} \mathrm{H} / \mathrm{H}$ using the rate of Ref. [6], used in the public version of PARThENoPE, and the best fit of Ref. [7] agree at the second decimal digit). Notice that this determination is lower than before, due to the order $1 \%$ increase of $\Omega_{b} h^{2}$ with respect to the Planck 2013 results. For a given baryon density value the theoretical uncertainty quoted above is dominated (more than $90 \%$ of the nuclear rate error contribution summed in quadrature) by the present error on experimental determination of the $d(p, \gamma)^{3} \mathrm{He}$ cross section; see Table I of Ref. [5].

This result is in agreement at $1-\sigma$ with the recent determination of Ref. [8] where, through a new analysis of all known deuterium absorption-line systems, it was found ${ }^{2} \mathrm{H} / \mathrm{H}=(2.53 \pm 0.04) \times 10^{-5}$ at $68 \%$ of C.L., but it is slightly larger. A possible way to get an even better agreement between the two values is to slightly decrease the effective neutrino number down to $N_{\text {eff }} \sim 2.84$. Another 
way is to increase the value of the $d(p, \gamma)^{3} \mathrm{He}$ astrophysical $S$ factor. This possibility was first explored in Ref. [9] and then analyzed in detail in Ref. [5], using the Planck 2013 data release, and in Ref. [3]. The conclusion of these studies is that increasing the $d(p, \gamma)^{3} \mathrm{He}$ thermal rate in the BBN temperature range by a factor of order $10 \%$ leads to a very good agreement between CMB anisotropy results and primordial deuterium abundance. Thus, a better determination of this $S$ factor with a reduction of the corresponding uncertainty in the BBN energy range, $E \simeq 30-300 \mathrm{keV}$, would be very important.

The astrophysical $S$ factor at low energy, around the solar Gamow peak $E_{G} \simeq 9 \mathrm{keV}$, is well known, thanks to the results of the LUNA experiment [10]. However, for the BBN relevant energy range, the experimental situation is rather unclear, since the only available experimental data [11] are quite in disagreement with the polynomial best fit of $S(E)$ for $E \simeq 0-2 \mathrm{MeV}$ [7]. This gives rise to an uncertainty on the cross section at the level of $6 \%-10 \%$. This is the main motivation of the experiment recently proposed by the LUNA Collaboration, with the goal of measuring the $d(p, \gamma)^{3} \mathrm{He}$ astrophysical $S$ factor in the BBN energy range with a $3 \%$ accuracy. A feasibility test has already been performed [12].

On the other hand, the $d(p, \gamma)^{3} \mathrm{He}$ astrophysical $S$ factor can be calculated using a microscopic ab initio approach. In fact, in Refs. [13,14] (see also references therein) the hyperspherical harmonics $(\mathrm{HH})$ technique was used to solve for the $A=3$ nuclear wave functions (WFs) using a realistic description of the nuclear interaction, which includes both two- and three-nucleon interactions. These are constructed to reproduce the $A=2$ large body of experimental data with a $\chi^{2} /$ datum $\sim 1$ (the Argonne $v_{18}$-AV18 - model [15]), and the $A=3$ binding energies (the Urbana IX-UIX-model [16]). Note that the HH method (see Ref. [17] for a review) is the only available one able to calculate the nuclear WF for the initial $p$ - $d$ scattering state at low energies, as the ones of interest here, including the Coulomb interaction between the charged initial particles. In the latest study of Ref. [14], a realistic model for the nuclear current operator was used, retaining both one- and many-body contributions. The latter are necessary in order to maintain gauge invariance in the presence of a system of interacting particles, and in Ref. [14] all the effort was put to construct these contributions which exactly satisfy the current conservation relation in conjunction with the AV18/UIX potential. The former, instead, was simply obtained performing a $1 / m$ expansion ( $m$ is the nucleon mass) of the singlenucleon covariant operator, and retaining the leading order contribution. The many-body contributions were found already in Ref. [13] essential to reach an excellent agreement with the LUNA data [10] around the solar Gamow peak $[7,14]$. In the energy range of interest for $\mathrm{BBN}$, on the other hand, the theoretical predictions of Ref. [14] were found to be $2 \%-10 \%$ higher than the central value for the polynomial fit of Ref. [7]. In the present Letter, our starting point is the work of Ref. [14], which, although very accurate, should be considered incomplete for two reasons: (i) no estimate of the theoretical uncertainty was given, in particular, that one arising from the solution of the $p-d$ scattering problem with the HH method; (ii) the one-body terms beyond the leading order operator, of the order $1 / \mathrm{m}^{3}$, were found a few years later [18], essential in order to get a reasonable agreement between theory and experiment for a related process, the $d(n, \gamma)^{3} \mathrm{H}$ radiative capture. Given the similarities between the $p-d$ and $n-d$ radiative captures, it is to be expected that these $1 / \mathrm{m}^{3}$ one-body contributions might be important also for the process here under consideration. The goal of the present Letter is to address the two above-mentioned issues and to verify whether the new prediction for the $d(p, \gamma)^{3} \mathrm{He}$ astrophysical $S$ factor goes in the direction of improving the consistency of theoretical BBN deuterium abundance prediction, the new Planck results, and the experimental data of Ref. [8]. We do not consider here ${ }^{4} \mathrm{He}$ primordial mass fraction $Y_{p}$, since it is insensitive to this reaction rate. For example, a change of the $d(p, \gamma)^{3} \mathrm{He} S$ factor by a factor of 2 affects $Y_{p}$ at the level of $0.04 \%$, too small to be appreciated with the present statistical and systematic uncertainties on its experimental determination; see, e.g., Ref. [19].

The present calculation.-We discuss here the two significant improvements in the calculation with respect to Ref. [14]. First of all, in the present work, we pay particular attention to the numerical determination of the $p-d$ WF. In particular, in each $L, S, J$ channel ( $L$ is the $p$ - $d$ orbital angular momentum, $S=1 / 2,3 / 2$ is the $p$ - $d$ total spin, and they are coupled so that $\mathbf{J}=\mathbf{L}+\mathbf{S}$ ) the WF $\Psi^{L S J}$ has been tested calculating $\langle H\rangle \equiv\left\langle\Psi^{L S J}|H| \Psi^{L S J}\right\rangle$ in a box with a radius of $70 \mathrm{fm}$, using a Monte Carlo method (independently on our technique to determine $\Psi$ ) and verifying that the correct result $\langle H\rangle=E-B_{d}$ is obtained within the requested accuracy (here $E$ is the $p$ - $d$ center-ofmass energy and $B_{d}$ the deuteron binding energy). Different grid points, dimensionality of the $\mathrm{HH}$ expansion basis, and values for the nonlinear parameter entering the polynomial expansion of the hyperradial functions (see Ref. [17]) have been checked in order to verify the above relation within $0.1 \%$. This procedure was not used in Ref. [14], and therefore the pattern of convergence for the WF (not the scattering matrix, i.e., the $p$ - $d$ phase shifts) was not verified. With this procedure we have been able to reduce the numerical uncertainty relative to the WFs in our astrophysical $S$ factor estimates of better than $1 \%$ for the whole energy range here under consideration (see Table I). Recall that the nuclear Hamiltonian used in the present study is the same as that of Ref. [14]; i.e., it retains the AV18/UIX potential model, which allows us to nicely reproduce, using the $\mathrm{HH}$ method, the ${ }^{3} \mathrm{He}$ binding energy and many $A=3$ scattering observables. In particular, in 
TABLE I. The $p$ - $d$ astrophysical $S$ factor (in $\mathrm{eV}$ b) for a representative set of energy values $E$ (in $\mathrm{keV}$ ). The theoretical percent uncertainty arising from the solution of the $p-d$ scattering problem with the $\mathrm{HH}$ method is given in the second column $\left(\Delta S_{W F}\right)$, while the additional contribution due to the one-body term of Eq. (1) $\left(\Delta \mathbf{j}^{(R C)}\right)$ is given in the last column, also in percent. Note that for $E=2 \mathrm{MeV}$ (last row), the value for $\Delta S_{W F}$ is below the permil level, and therefore not quoted.

\begin{tabular}{lccc}
\hline \hline$E[\mathrm{keV}]$ & $S(E)[\mathrm{eV} \mathrm{b}]$ & $\Delta S_{W F}[\%]$ & $\Delta \mathbf{j}^{(R C)}[\%]$ \\
\hline 10 & 0.286 & 0.1 & +0.8 \\
20 & 0.355 & 1.0 & +1.1 \\
35 & 0.460 & 1.1 & +1.3 \\
50 & 0.570 & 0.9 & +1.7 \\
70 & 0.716 & 0.4 & +2.1 \\
95 & 0.912 & 0.3 & +2.3 \\
120 & 1.112 & 0.8 & +2.4 \\
145 & 1.317 & 0.4 & +2.5 \\
170 & 1.529 & 0.4 & +2.6 \\
195 & 1.748 & 0.4 & +2.6 \\
220 & 1.968 & 0.5 & +2.8 \\
245 & 2.197 & 0.4 & +2.7 \\
260 & 2.343 & 0.9 & +2.8 \\
300 & 2.716 & 0.5 & +2.7 \\
400 & 3.676 & 0.6 & +2.7 \\
500 & 4.739 & 0.2 & +2.7 \\
750 & 7.539 & 0.3 & +2.6 \\
1000 & 10.685 & 0.4 & +2.7 \\
2000 & 25.908 & $\ldots$ & +2.3 \\
\hline \hline
\end{tabular}

$p$ - $d$ elastic scattering, the total cross section available data are nicely reproduced. Some discrepancies appear only for few delicate polarization observables (see Fig. 16 of Ref. [20]). Furthermore, all partial waves with $J \leq 5 / 2$ and both parities have been retained.

The new term of the nuclear electromagnetic (EM) current here included is that arising from the $1 / m$ expansion of the single-nucleon covariant current operator, and is a relativistic correction of the order $1 / \mathrm{m}^{3}$. It has been derived in Refs. [21,22] in the context of chiral effective field theory, and can be written as [21]

$$
\begin{aligned}
\mathbf{j}_{i}^{R C}= & -\frac{e e_{i}}{8 m^{3}}\left[2\left(K_{i}^{2}+q^{2} / 4\right)\left(2 \mathbf{K}_{i}+\mathrm{i} \boldsymbol{\sigma}_{i} \times \mathbf{q}\right)\right. \\
& \left.+\mathbf{K}_{i} \cdot \mathbf{q}\left(\mathbf{q}+2 \mathrm{i} \boldsymbol{\sigma}_{i} \times \mathbf{K}_{i}\right)\right]-\frac{\mathrm{i} e \kappa_{i}}{8 m^{3}}\left[\mathbf{K}_{i} \cdot \mathbf{q}\left(4 \boldsymbol{\sigma}_{i} \times \mathbf{K}_{i}-\mathbf{i} \mathbf{q}\right)\right. \\
& \left.-\left(2 \mathrm{i} \mathbf{K}_{\mathbf{i}}-\boldsymbol{\sigma}_{i} \times \mathbf{q}\right) q^{2} / 2+2\left(\mathbf{K}_{\mathbf{i}} \times \mathbf{q}\right) \boldsymbol{\sigma}_{i} \times \mathbf{K}_{i}\right]
\end{aligned}
$$

where $\mathbf{K}_{i}=\left(\mathbf{p}_{i}^{\prime}+\mathbf{p}_{i}\right) / 2, \mathbf{p}_{i}$ and $\mathbf{p}_{i}^{\prime}$ being the initial and final momenta of the nucleon, $\mathbf{q}$ is the photon momentum, $e$ is the electron charge, $e_{i}=\left(1+\tau_{i, z}\right) / 2$, the charge-projection isospin operator, $\kappa_{i}=\left(\kappa_{S}-\kappa_{V} \tau_{i, z}\right) / 2, \quad \kappa_{S}=$ $-0.12 \mu_{N}\left(\kappa_{V}=3.706 \mu_{N}\right)$ being the isoscalar (isovector) combination of the anomalous magnetic moments of proton and neutron, and $\sigma_{i}\left(\boldsymbol{\tau}_{i}\right)$ are the spin (isospin) Pauli matrices. It was found in Ref. [18] that $\mathbf{j}_{i}^{R C}$ reduces the $n-d$ total cross section at thermal energies of about $4 \%-5 \%$, bringing the theoretical prediction in a much better agreement with the experimental datum (within 4\%). In the $p$ - $d$ case, instead, we have found that the operator $\mathbf{j}_{i}^{R C}$ gives a positive contribution, increasing the astrophysical $S$ factor of $1 \%-3 \%$ over the whole energy range considered here (see Table I).

The astrophysical $S$ factor obtained in the present work is listed in Table I and plotted in Fig. 1, where it is compared with the previous calculation of Ref. [14], with the existing data of Refs. [10,11,23,24], and with the polynomial fit of Ref. [7]. To be noticed that the theoretical uncertainty arising from the solution of the $p$ - $d$ scattering problem with the $\mathrm{HH}$ method are not visible on the plot, although the corresponding symbols retain an error. The present results are systematically larger (about 8\%-10\%) than those of Ref. [14]. We have investigated the origin of such an increase, and we have found that only $1 \%-3 \%$, depending on the energy value, is due to the one-body $1 / \mathrm{m}^{3}$ contribution. Therefore, the remaining $5 \%-8 \%$ is due to the new solutions of the $A=3$ (scattering) problem. In fact,

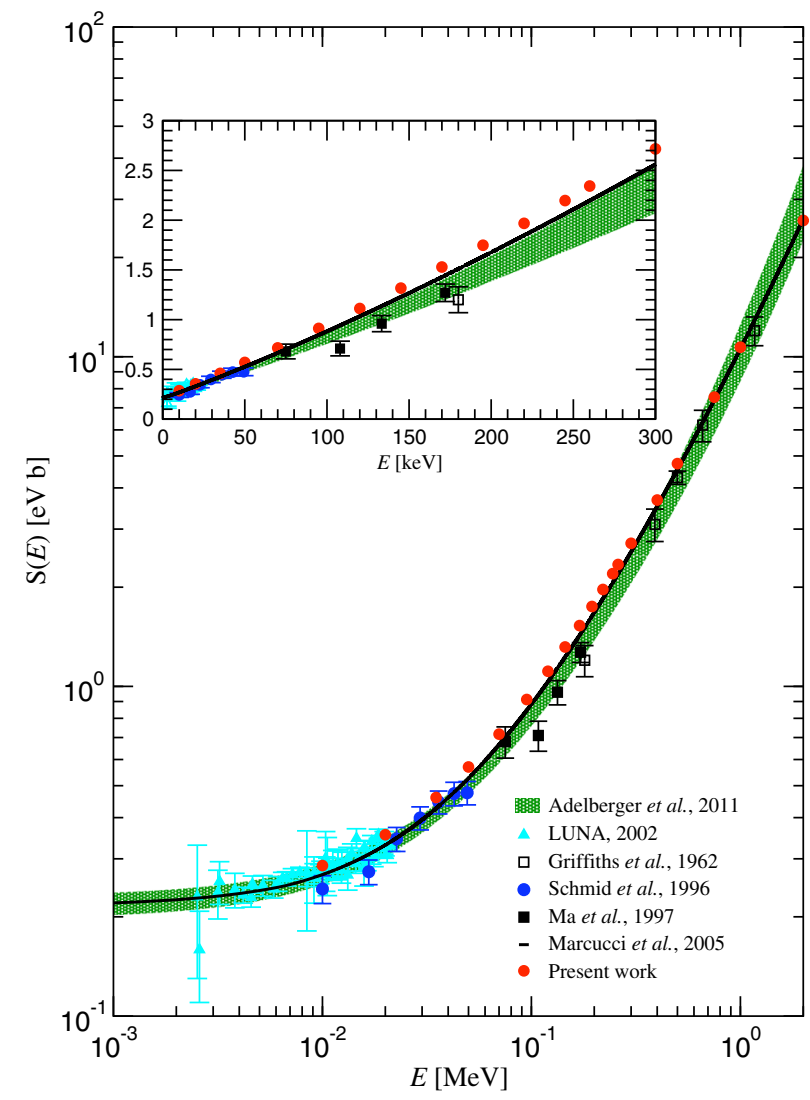

FIG. 1. The astrophysical $S$ factor obtained in the present work (red points) is plotted together with the available experimental data of Refs. [10,11,23,24], the calculation of Ref. [14] (solid black line), and the quadratic best fit to the data of Ref. [7] (green band). The inset shows the astrophysical $S$ factor in the 0-300 keV energy range, since the relevant $\mathrm{BBN}$ energy range is $30-300 \mathrm{keV}$. 
the present WFs have been obtained with the same $\mathrm{HH}$ technique as in Ref. [14], but with the goal of reaching the required higher accuracy dictated by BBN, and thus they have been tested one by one, as explained above.

Implications for BBN.-To study the effect of the new $a b$ initio determination of the $d(p, \gamma)^{3} \mathrm{He} S$ factor on primordial deuterium produced during $\mathrm{BBN}$ we have computed the corresponding thermal rate using the best fit values reported in the second column of Table I and modified the numerical code PARTHENOPE [4] accordingly. The new rate in the $\mathrm{BBN}$ temperature range $(0.01-0.1 \mathrm{MeV})$ is larger by a factor $1.12-1.15$ with respect to what is found using the $S$ factor of Ref. [7]. The theoretical results for deuterium to hydrogen density ratio ${ }^{2} \mathrm{H} / \mathrm{H}_{\mathrm{th}}$ are then computed as a function of two parameters, the baryon density $\Omega_{b} h^{2}$ and $N_{\text {eff }}$, and compared with the experimental determination ${ }^{2} \mathrm{H} / \mathrm{H}_{\exp }$ of Ref. [8]. To obtain the best fit values and uncertainty on these parameters we then consider the likelihood function

$$
\begin{aligned}
\mathcal{L}\left(\Omega_{b} h^{2}, N_{\text {eff }}\right)= & \mathcal{L}_{\text {Planck }}\left(\Omega_{b} h^{2}\right) \\
& \times \exp \left(-\frac{\left({ }^{2} \mathrm{H} / \mathrm{H}_{\mathrm{th}}\left(\Omega_{b} h^{2}, N_{\text {eff }}\right)-{ }^{2} \mathrm{H} / \mathrm{H}_{\text {exp }}\right)^{2}}{2\left(\sigma_{\text {exp }}^{2}+\sigma_{\text {th }}^{2}\right)}\right),
\end{aligned}
$$

where $\sigma_{\text {exp }}=0.04$ as obtained in Ref. [8]. $\mathcal{L}_{\text {Planck }}\left(\Omega_{b} h^{2}\right)$ is a Gaussian prior corresponding to the Planck result of Ref. [3], while $\sigma_{\text {th }}$ is the propagated error on deuterium yield due to the present experimental uncertainty on other leading nuclear reactions relevant for deuterium production and destruction during $\mathrm{BBN}$, namely, $d(d, n)^{3} \mathrm{He}$ and $d(d, p)^{3} \mathrm{H}$. It also accounts for the $d(p, \gamma)^{3} \mathrm{He} S$ factor theoretical uncertainty of Table I, and the small error on the $p(n, \gamma) d$ rate, both very subdominant with respect to the other error sources.

For the Planck 2015 value of $\Omega_{b} h^{2}$ and standard $N_{\text {eff }}$ we get ${ }^{2} \mathrm{H} / \mathrm{H}_{\mathrm{th}}=(2.49 \pm 0.03 \pm 0.03) \times 10^{-5}$, where the two errors are due to nuclear rate and $\Omega_{b} h^{2}$ uncertainties, respectively. In Fig. 2 we show the likelihood contours (68, 95, and 99\% C.L.) in the $\Omega_{b} h^{2}-N_{\text {eff }}$ plane. The Planck 2015 best fit results and 68\% C.L. error bars are also shown. The agreement is very good, within 1- $\sigma$. Marginalizing over baryon energy density, we find $N_{\text {eff }}=3.18 \pm 0.16(0.32)$, where the error is at $68 \%$ (95\%) C.L., which is fully consistent with a standard radiation content during BBN. Notice that, once the Planck 2015 prior on $\Omega_{b} h^{2}$ is used, the uncertainty one gets on $N_{\text {eff }}$ from ${ }^{2} \mathrm{H} / \mathrm{H}$ alone is of the same order of magnitude obtained from $\mathrm{CMB}$, once baryon acoustic oscillation data are also exploited. In this case, the Planck result is $N_{\text {eff }}=3.04 \pm 0.18$ (68\% C.L.) [3].

Summary and outlook.-The astrophysical $S$ factor for the $d(p, \gamma)^{3} \mathrm{He}$ reaction is crucial to determine the consistency of the BBN theoretical prediction for deuterium

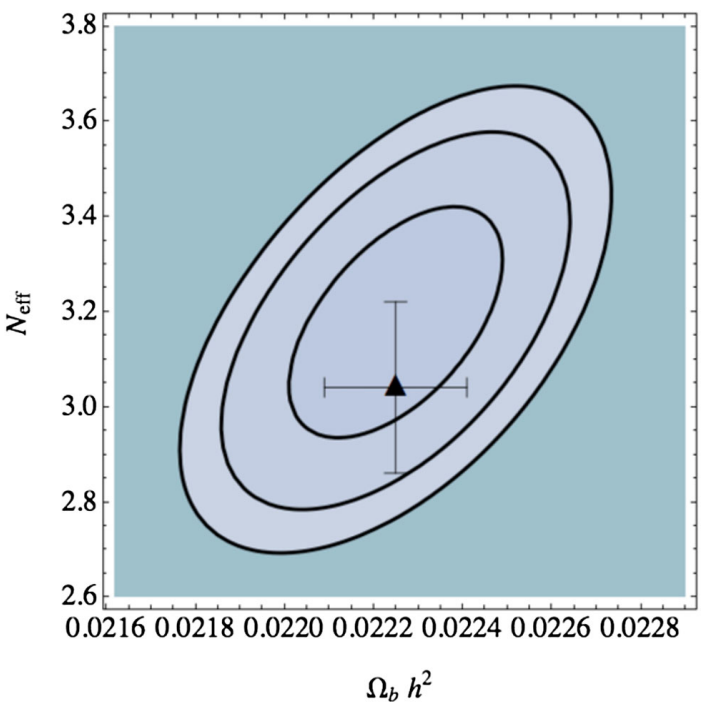

FIG. 2. The likelihood contours $(68,95$, and $99 \%$ C.L.) in the $\Omega_{b} h^{2}-N_{\text {eff }}$ plane from ${ }^{2} \mathrm{H} / \mathrm{H}$, with the Planck 2015 prior on $\Omega_{b} h^{2}$, a free $N_{\text {eff }}$, and using the experimental result of Ref. [8]. The triangle is the best fit value of Planck 2015 results for these parameters, with corresponding $68 \%$ C.L. error bars [3].

abundance, the new Planck results, and the most recent experimental determination of such abundance [8]. In the absence of an accurate experimental determination in the energy range of interest for $\mathrm{BBN}, 30-300 \mathrm{keV}$, we have performed a new theoretical $a b$ initio calculation, using the most up-to-date techniques to calculate the WFs for the initial scattering and final bound states, with the realistic AV18/UIX potential model, as well as the most up-to-date realistic model for the nuclear current operator, which satisfies gauge invariance with the adopted Hamiltonian and retains the $1 / \mathrm{m}^{3}$ contribution in the one-body operator. We have found that the numerical uncertainty relative to the WF in the $S$ factor is lower than $1 \%$, while the $1 / \mathrm{m}^{3}$ onebody contribution increases the $S$ factor by $1 \%-3 \%$ over the whole energy range. We have then investigated the effect of this new $a b$ initio determination on the primordial deuterium abundance. We find that $\mathrm{BBN}$ predictions are in very good agreement with the Planck 2015 results and the experimental result of Ref. [8]. Also, the inferred value for $N_{\text {eff }}$ is fully consistent with a standard radiation content during BBN. Of course, our results ought to be confirmed by direct measurement of the $d(p, \gamma)^{3} \mathrm{He} S$ factor, as it is planned at the Gran Sasso National Laboratories (Italy), by the LUNA Collaboration. Such a measurement will therefore turn out to be crucial in this context. Finally, it should be mentioned that a theoretical ab initio calculation of this process is at reach also within the so-called chiral effective field theory $(\chi \mathrm{EFT})$ framework, which provides a direct connection between quantum chromodynamics and the strong and electroweak interactions in nuclei, and is a practical calculational scheme, which can be improved systematically. The first studies along this line have been 
done for the EM structure of light nuclei $[25,26]$. At present, however, the consistency between the $\chi \mathrm{EFT}$ nuclear potentials and EM currents necessary to satisfy exactly gauge invariance (as in the case of the calculation presented here) has not been yet achieved, making the $\chi$ EFT results not completely reliable at the accuracy level necessary for BBN predictions. Work on this issue, though, is currently underway.

The authors acknowledge the support of the computer center staff at INFN-Pisa, where part of the present calculations were performed. G. M. acknowledges support by INFN I. S. TASP and PRIN "Fisica Astroparticellare: Neutrini ed Universo Primordiale" of the Italian Ministero dell'Istruzione, Università e Ricerca. L. E. M. would like to thank the LUNA Collaboration for triggering the present study and for useful discussions.

Note added in proof.-After completing this work a reevaluation of the $d(p, \gamma)^{3} \mathrm{He}, \quad d(d, n)^{3} \mathrm{He}$, and $d(d, p)^{3} \mathrm{H}$ reaction rates has been also presented in Ref. [27].

[1] F. Iocco, G. Mangano, G. Miele, O. Pisanti, and P. D. Serpico, Phys. Rep. 472, 1 (2009).

[2] G. Mangano, G. Miele, S. Pastor, T. Pinto, O. Pisanti, and P. D. Serpico, Nucl. Phys. B729, 221 (2005).

[3] P. A. R. Ade et al. (Planck Collaboration), arXiv:1502.01589.

[4] O. Pisanti, A. Cirillo, S. Esposito, F. Iocco, G. Mangano, G. Miele, and P. D. Serpico, Comput. Phys. Commun. 178, 956 (2008).

[5] E. Di Valentino, C. Gustavino, J. Lesgourgues, G. Mangano, A. Melchiorri, G. Miele, and O. Pisanti, Phys. Rev. D 90, 023543 (2014).

[6] P. D. Serpico, S. Esposito, F. Iocco, G. Mangano, G. Miele, and O. Pisanti, J. Cosmol. Astropart. Phys. 12 (2004) 010.

[7] E. G. Adelberger et al., Rev. Mod. Phys. 83, 195 (2011).

[8] R. Cooke, M. Pettini, R. A. Jorgenson, M. T. Murphy, and C. C. Steidel, Astrophys. J. 781, 31 (2014).
[9] K. M. Nollett and G. P. Holder, arXiv:1112.2683.

[10] C. Casella et al. (LUNA Collaboration), Nucl. Phys. A706, 203 (2002).

[11] L. Ma, H. J. Karwowski, C. R. Brune, Z. Ayer, T. C. Black, J. C. Blackmon, E. J. Ludwig, M. Viviani, A. Kievsky, and R. Schiavilla, Phys. Rev. C 55, 588 (1997).

[12] D. Trezzi for the LUNA Collaboration, private communication.

[13] M. Viviani, A. Kievsky, L. E. Marcucci, S. Rosati, and R. Schiavilla, Phys. Rev. C 61, 064001 (2000).

[14] L. E. Marcucci, M. Viviani, R. Schiavilla, A. Kievsky, and S. Rosati, Phys. Rev. C 72, 014001 (2005).

[15] R. B. Wiringa, V. G. J. Stoks, and R. Schiavilla, Phys. Rev. C 51, 38 (1995).

[16] B. S. Pudliner, V. R. Pandharipande, J. Carlson, S. C. Pieper, and R. B. Wiringa, Phys. Rev. C 56, 1720 (1997).

[17] A. Kievsky, S. Rosati, M. Viviani, L. E. Marcucci, and L. Girlanda, J. Phys. G 35, 063101 (2008).

[18] L. Girlanda, A. Kievsky, L. E. Marcucci, S. Pastore, R. Schiavilla, and M. Viviani, Phys. Rev. Lett. 105, 232502 (2010).

[19] R. H. Cyburt, B. D. Fields, K. A. Olive, and T. H. Yeh, arXiv:1505.01076 [Reviews of Modern Physics (to be published)].

[20] L. E. Marcucci, A. Kievsky, L. Girlanda, S. Rosati, and M. Viviani, Phys. Rev. C 80, 034003 (2009).

[21] S. Pastore, L. Girlanda, R. Schiavilla, M. Viviani, and R. B. Wiringa, Phys. Rev. C 80, 034004 (2009).

[22] Y.-H. Song, R. Lazauskas, and T.-S. Park, Phys. Rev. C 79, 064002 (2009).

[23] G. M. Griffiths, M. Lal, C. D. Scarfe, Can. J. Phys. 41, 724 (1963).

[24] G. J. Schmid et al., Phys. Rev. Lett. 76, 3088 (1996).

[25] M. Piarulli, L. Girlanda, L. E. Marcucci, S. Pastore, R. Schiavilla, and M. Viviani, Phys. Rev. C 87, 014006 (2013).

[26] L. E. Marcucci, F. Gross, M. T. Peña, M. Piarulli, R. Schiavilla, I. Sick, A. Stadler, J. W. Van Orden, and M. Viviani, J. Phys. G 43, 023002 (2016).

[27] A. Coc, P. Petitjean, J. P. Uzan, E. Vangioni, P. Descouvemont, C. Illiadis, and R. Longland, Phys. Rev. D 92, 123526 (2015). 